\title{
A Fuzzy Logic System for Admissibility of Prospective Student to Nursery Class
}

\author{
Goldendeep Kaur \\ CSE Department \\ GNDU RC Jalandhar
}

\author{
Prabhjot Singh \\ ECE Department \\ Chandigarh University Gharuan
}

\begin{abstract}
Education plays a vital role in our lives. In today's constantly changing technological world, education is necessity after food, clothing and shelter. Competition prevails in every sphere of life. Even at primary level there is massive rush for admissions. Every school has its own criteria for selecting prospective students. This research is an attempt to design and implement a fuzzy logic system to identify the eligibility of the concerned students. In the system designed, four input parameters which are Neighborhood points, Educational Qualification of Parents, Siblings points and Alumni points are evaluated using Fuzzy system to infer an output parameter eligibility according to which we can decide whether the child is eligible for admission.
\end{abstract}

\section{Keywords}

Fuzzy Logic, Eligibility Criteria, Admissions, Fuzzy Rules.

\section{INTRODUCTION}

Our modern economy and culture has changed with competition entering in every sphere of life. There is an insane rush to have the best things in life. Even in case of education competition prevails for the best schools and colleges. People want their children to go to the best schools, best colleges and universities and get the best jobs. There are a vast number of students for the organizations to choose from. Hence there is a dire need for a fair system which should be transparent and clear for the selection of students to schools and colleges so that there is no partiality regarding the selection. People are very much concerned about which schools their children should go as nursery is the stepping stone for the children as they begin their long journey through childhood, grow up through various stages of education. Due to the increased pressure on the schools to choose the students from a large pool has led to partiality in the selection process. The State of Delhi has to face many problems such as favoring only a particular community of people, asking for donations from the parents for the school, not following the guidelines given by ministry of HRD etc. The state government has to step in to solve this problem so that every child should get equal opportunity to study in the best schools and that should be based on the fair selection process. This research is an attempt to design system based on which the eligibility of the student can be decided. It has been found that there are various parameters depending on which decision regarding selection is made. These include:

- Neighborhood points

- Educational Qualification of Parents

- Siblings' points

- Alumni points

- Children with special needs

- School-specific parameters
The paper is organized as follows: Section 2 describes introduction to fuzzy logic, Section 3 describes the fuzzy inference engine, Section 4 describes the simulation architecture and Section 5 concludes the paper.

\section{FUZZY LOGIC}

FUZZY logic was proposed in 1965. In this context, fuzzy logic is a problem-solving control system methodology that can be put into effect in hardware, software, or a combination of both. It is a form of multi-valued logic that deals with approximation rather than exact values. Fuzzy provides a remarkably simple way to draw definite conclusions from vague, obscure or non-specific information. In a sense, fuzzy logic resembles human decision making with its ability to work from approximate data and find precise solutions.[2] Fuzzy logic subsumes a straightforward, rule-based IF $X$ AND Y THEN $\mathrm{Z}$ technique to a solving control problem rather than experimenting to model a system mathematically. Fuzzy logic variables may have a truth value that ranges in between 0 and 1 . It requires numerical parameters in order to function such as what is considered significance error and significant rate-of-change-of-error, but precise values of these numbers are usually not crucial unless very responsive performance is required.

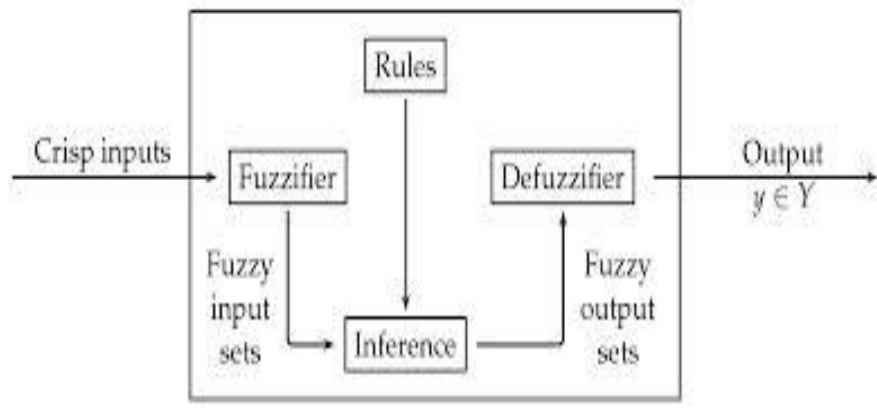

Fig 1: Fuzzy Logic architecture

\section{FUZZY INFERENCE ENGINE}

A Fuzzy inference engine is an artificial intelligence tool based upon fuzzy set theory and if-then rules. Its basic structure consists of three conceptual components: a rule base which contains selection of fuzzy rules, a data base which defines membership functions used in fuzzy rules and a reasoning mechanism which performs inference procedures upon the rules and given facts to derive at a reasonable output or conclusion. It is a way of mapping an input space to an output space. [9] It is implemented in two phases given below.

\subsection{Fuzzification}

Fuzzification is a method of mapping measured input values fuzzy membership functions. A membership function is a curve that describes how each position in the input space is mapped to a membership value within 0 and 1 . There are 
distinct shapes of membership functions; triangular, piecewise, trapezoid, Gaussian, bell-shaped, etc. [4]

\subsection{Defuzzification}

Defuzzification is a conversion of internal fuzzy output variables into crisp values that can actually be used. It is done after the evaluation of inputs and applies them to the rule base.

\section{SIMULATION ARCHITECTURE}

The simulation model is built with the Fuzzy Logic Toolbox of Math Works. Fuzzification comprises the process of transforming crisp value into grade of membership for linguistic terms of fuzzy sets. The first step involves identification of the parameters that are to be fuzzified and determining their respective range of values. The inference after the interaction is the value for each performance parameter.

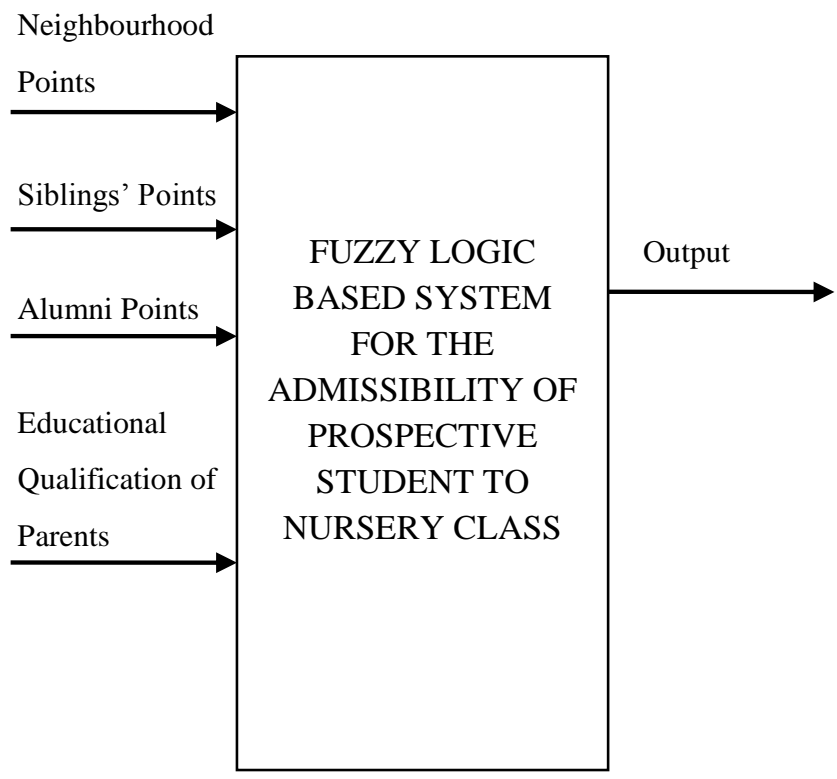

Fig 2: Fuzzy Logic Based System

\subsection{Input and Output Parameters}

The input and output parameters are created in FIS editor as shown in the Fig 2. We have considered four input parameters and one output applied to the FIS.

Input parameters are:

- Neighborhood points

- $\quad$ Siblings points

- Alumni points

- Educational Qualification of Parents

There is one output parameter as shown, which is Eligibility.

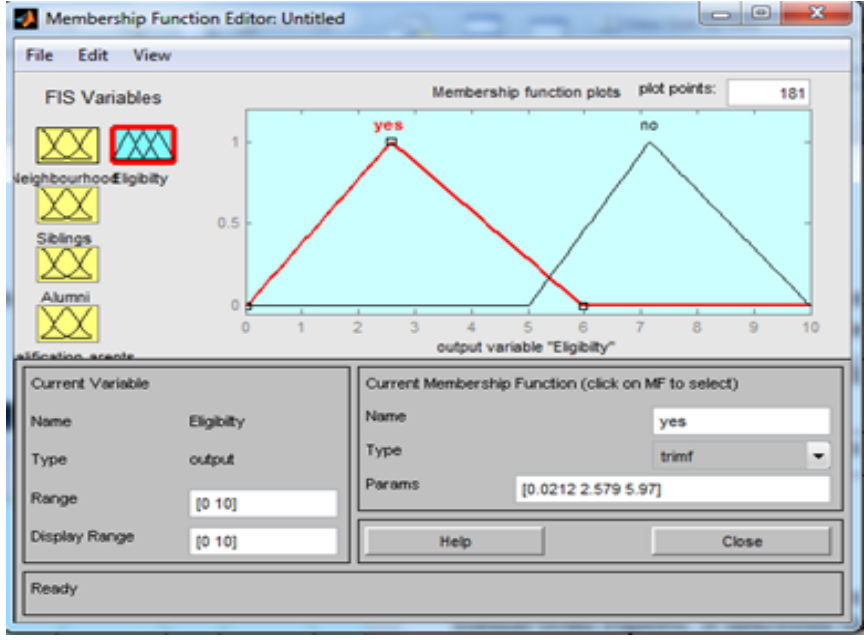

Fig 3: Input and Output Parameters

\subsection{Membership Functions}

The membership function editor is used to define the properties of the membership function for the systems variables. It is a graphical representation of the magnitude of participation of each input and associates each weighting with the inputs that is later processed. The rules and the input membership values are used in a combined manner to determine their influence on the final output conclusion. The defuzzified crisp output which drives the system is obtained, once the functions are scaled and combined.

Table I. Membership functions for given input parameters

\begin{tabular}{|l|l|l|l|}
\hline Input parameters & Mf1 & Mf2 & Mf3 \\
\hline Neighborhood points & Low & Medium & High \\
\hline Siblings points & Less & Medium & More \\
\hline Alumni points & Less & Medium & More \\
\hline $\begin{array}{l}\text { Educational Qualification } \\
\text { of Parents }\end{array}$ & Low & Medium & High \\
\hline
\end{tabular}

Table II. Membership functions for given output parameters:

\begin{tabular}{|l|l|l|}
\hline Output parameter & Mf1 & Mf2 \\
\hline Eligibility & Yes & No \\
\hline
\end{tabular}

\subsection{Rule Base}

A fuzzy rule base is a collection of knowledge in the If-Then format from experts. It describes the relationship between fuzzy input parameters and output. As per the input and output parameters fuzzified, rule base is generated by applying reasoning to decide the eligibility of a student. Various rules have been generated to train the fuzzy system during the implementation part.

While formulating the if-then rules, a specific hierarchy on the basis of priority has been followed. Among all the four parameters, Neighborhood points have been given the top priority followed by Siblings points. The least importance in the rules has been given to Educational Qualification of Parents. This system is basically designed for non minority schools where there is no quota. Fig 4 shows rule viewer and 
Fig 5 shows surface viewer for our system. Fig 6 shows the .m file to obtain system results and Fig 7 shows the inference calculated based on these rules.

Sample rules have been shown.

- If Neighborhood points are LOW, Siblings points are MORE, Alumni points are MEDIUM, Qualification of Parents is MEDIUM then Eligibility is YES.

- If Neighborhood points are MEDIUM, Siblings points are MORE, Alumni points are MEDIUM, Qualification of Parents is LOW then Eligibility is YES.

- If Neighborhood points are HIGH, Siblings points are LESS, Alumni points are MEDIUM, Qualification of Parents is LOW then Eligibility is NO.

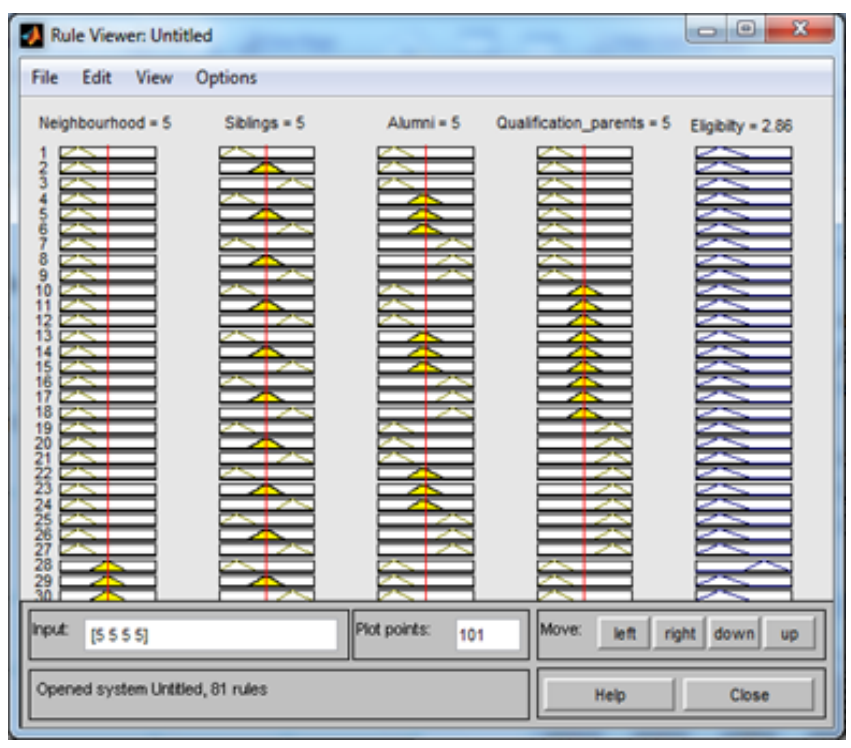

Fig 4: Rule viewer

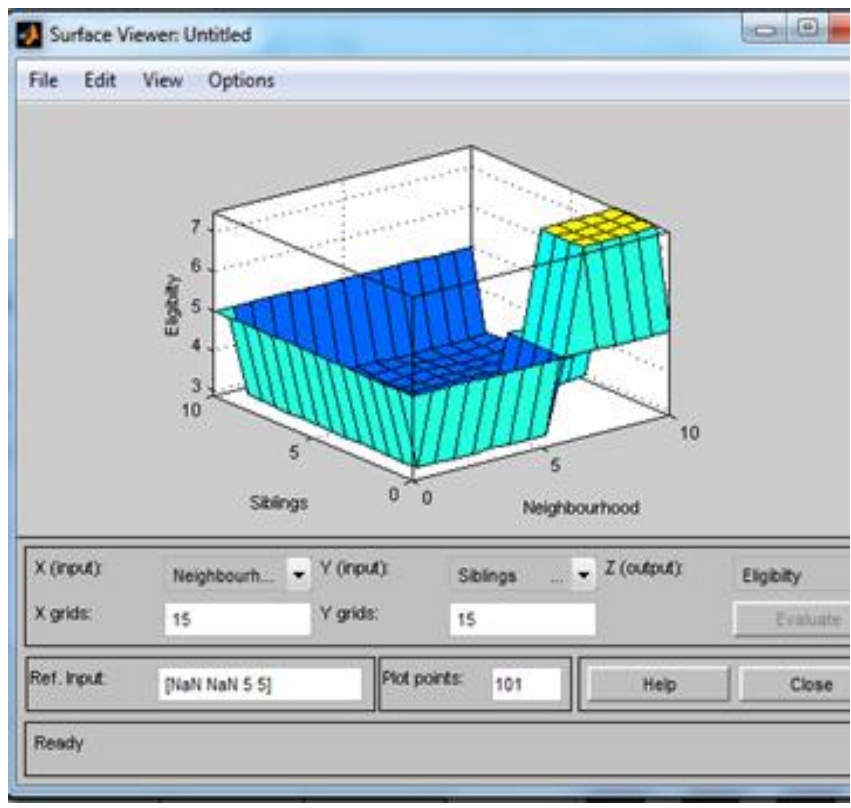

Fig 5: Surface Viewer (Neighborhood points vs. Siblings points)

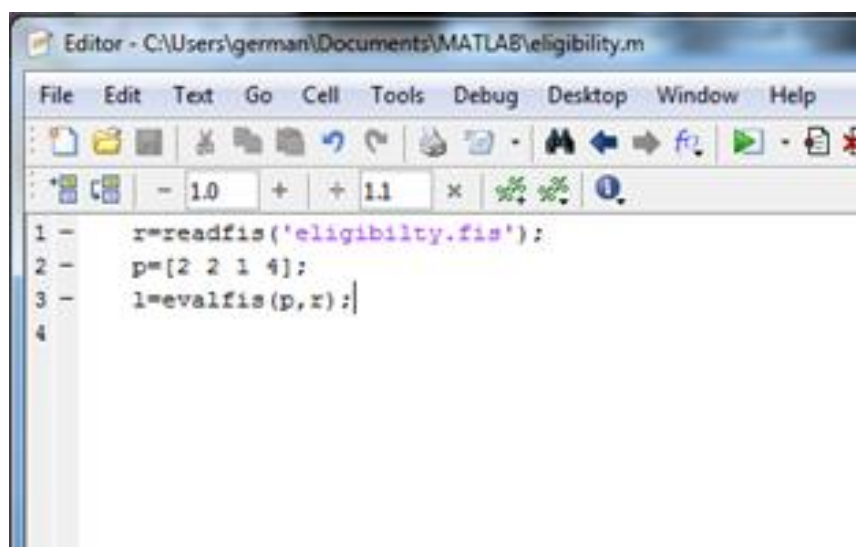

Fig 6: .m file to obtain the system results

\begin{tabular}{|c|c|c|c|c|}
\hline & \multicolumn{4}{|l|}{ Input variables } \\
\hline NP & SP & AP & QoP & Eligibility \\
& & & & \\
\hline 2 & 2 & 1 & 4 & 2.9024 \\
\hline 7 & 2 & 1 & 4 & 7.4267 \\
\hline 7 & 7 & 5 & 8 & 2.8968 \\
\hline 3 & 7 & 3 & 2 & 2.9160 \\
\hline 8 & 2 & 3 & 2 & 7.4382 \\
\hline 5 & 6 & 5 & 7 & 2.9072 \\
\hline 9 & 2 & 2 & 4 & 7.4350 \\
\hline 10 & 5 & 6 & 5 & 3 \\
\hline
\end{tabular}

Fig 7: Inference Table

\section{CONCLUSION}

This system of determining the eligibility of a prospective student is a very effective procedure that makes use of fuzzy logic to solve real world problems. The proposed system solves the problems of thousands of parents and children who have to face uncertainty and frustration every year. Education is vital in our lives and the beginning of school is the first stepping stone. Thus a model has been designed as a decision support system which can make matter easier and transparent for the authorities and parents. The membership functions and fuzzy rule bases have been developed based on logical reasoning after extensive survey. The results obtained reflect that the proposed system can be used to determine the eligibility of a student. This research can later be extended by considering remaining parameters after reaching in to more surveys for further improvements.

\section{REFERENCES}

[1] Ekong Victor., Ekong Uyinomen., Uwadiae Enobakhare.: A Fuzzy Inference System for predicting depression risk levels. Emmanuel African Journal of Mathematics and Computer Science Research, Vol. 6(10), pp 197-204 (2013).

[2] G.A Bhosle., R.S. Kamath.: Fuzzy inference system for teaching staff performance appraisal. International 
journal of computer and information technology (ISSN 2279-0764) volume 02-Issue (2013).

[3] José Luis Aznarte M., José Manuel Benítez., Juan Luis Castro.,: Smooth transition autoregressive models and fuzzy rule-based systems: Functional equivalence and consequences. Received (28 March 2006); received in revised form (1 March 2007); accepted (24 March 2007) Available online 7 April 2007, http://sci2s.ugr.es/publications/ficheros/2007-aznarte$\underline{\text { FSS.pdf }}$

[4] J.-S.R.Jang.: Adaptive-Network-Based Fuzzy Inference System. In proceedings of IEEE Transactions on Systems, Man, and Cybernetics, Vol. 23, No. 3(1993).

[5] Lazim Abudullah1., Mohd Nordin Abd Rahman.: Employee likelihood of purchasing health insurance using fuzzy inference system. International Journal of Computer Science Issues. Vol 9, Issue 1, No. 2 (2012).

[6] Maltab Guide.

[7] Maedeh Rasoulzadeh.: facial expression recognition using fuzzy inference system. International Journal of Engineering andInnovative Technology. Vol. 1, Issue 4 (2012).

[8] Steven D. Kaehler.: Fuzzy Logic Tutorial. Retrieved from:http://www.seattlerobotics.org/encoder/mar98/fuz/f lindex.html.

[9] Zalinda Othman, Khairanun Subari., Norhashimah Morad.: Application of fuzzy inference systems and genetic algorithms in integrated process process planning and scheduling. Academin Staff Training Research, Research Grant No. 305/PTEKIND/622140. 